\title{
Performance Analysis for a Guaranteed Handover Service in an LEO Constellation with a "Satellite-Fixed Cell" System
}

\author{
Gérard Maral, Joaquin Restrepo, Enrico Del Re, Senior Member, IEEE, \\ Romano Fantacci, Senior Member, IEEE, and Giovanni Giambene, Member, IEEE
}

\begin{abstract}
It is anticipated that the satellite component of the future universal mobile telecommunications system (UMTS) will be based (partly or totally) on non-GEOstationary (nonGEO) constellations of satellites to serve mixed populations of users, each category being treated through different contracts stipulating different quality of service (QoS). In particular, we envisage a high-quality premium service which guarantees the success of each handover procedure, called guaranteed handover (GH) service, and a low-cost lower quality service called regular service, where handover failures are accepted provided that the probability of a call being unsuccessful does not exceed a given value. This paper proposes a strategy which eliminates forced call terminations due to handover failures, thus allowing the GH service. This procedure applies to low earth orbit (LEO) constellations using the satellite-fixed cell technique. An analytical model has been derived to calculate QoS parameters for a mixed population of GH and regular users. Providing both GH service to some users and regular service to other users requires an increased satellite capacity with respect to the case where all the users are served with the regular service; this capacity increase has been evaluated as a function of the percentage of $\mathbf{G H}$ users, the traffic load per cell, and the considered satellite mobility environment. The GH approach has been validated through the comparison with another scheme which envisages the queuing of handover requests for privileged users.
\end{abstract}

Index Terms - Personal communication networks, satellite communications.

\section{INTRODUCTION}

$\mathbf{T}$ IHE UNIVERSAL mobile telecommunications system (UMTS) represents the realization of a new generation of mobile communications technology which aims at providing new and personalized services [1]. One of the main objectives of UMTS is to offer the same range of services as provided by fixed communications networks and, possibly, with the same quality. This paper is concerned with the satellite component of the future UMTS that will be based partly or totally on non-GEOstationary (non-GEO) constellations of satellites [i.e., low earth orbit (LEO) and medium earth orbit (MEO)] [2]. Given the allocated bandwidth, the capacity of the network

Manuscript received January 12, 1998; revised July 8, 1998.

G. Maral is with the Ecole Nationale Supérieure des Télécommunications (ENST), Toulouse, France.

J. Restrepo is with the Universidad Pontificia Bolivariana and COLCIENCIAS, Colombia.

E. Del Re, R. Fantacci, and G. Giambene are with the Dipartimento di Ingegneria Elettronica, Università degli Studi di Firenze, Firenze, Italy.

Publisher Item Identifier S 0018-9545(98)08271-1. can be increased through frequency reuse not only among satellite footprints (=satellite coverage areas), but also within the footprints themselves [3]. This is achieved by dividing the footprint into cells, each one corresponding to a specific beam of the satellite antenna radiation pattern. With satellitefixed cell systems, beams maintain a constant geometry with respect to the spacecraft, and the cells on the ground move along with the satellite [2]. With earth-fixed cell systems, the antenna beams are steered so as to point toward a given cell on the earth during some time interval [2], [4]. This paper only deals with satellite-fixed cell systems.

Due to the satellite motion with respect to the earth's surface, an active user terminal may change beam, and eventually satellite, while a call is in progress. The transfer of an ongoing call from one cell to the next one is named beam handover, and the transfer from a satellite to the next one is named satellite handover [2]. In non-GEO satellite systems, the handover rate is conditioned by the satellite velocity (and therefore by the satellite constellation altitude) and not by that of the user on the earth's surface [5]. Thus, calls will experience both beam handovers and satellite handovers, regardless of whether the users are fixed or mobile. The handover may fail as a result of the incoming cell having no idle channel. Such a handover failure results in a forced termination of the ongoing call [6]. This will affect both fixed and mobile users. Forced termination can also be caused by propagation impairments.

Forced termination of an ongoing call is perceived by the user as a frustrating event, and the system designer should therefore aim at achieving a low forced-termination probability. Current terrestrial cellular networks and GEO systems are typically designed to provide a call forced-termination probability of about $1 \%$, but even more stringent requirements have to be considered [7]. In these systems, users at fixed locations (i.e., fixed users) do not experience handover failures, whereas in non-GEO systems they could have unsuccessful handover procedures due to the satellite motion. Consequently, it makes sense to envisage that some fixed users will require a high quality of service (QoS) when subscribing to services provided by non-GEO satellite systems. In addition to this, some mobile users could also be interested in a high-quality service via satellite.

In order to meet the expectation of such potential users, it is necessary to combat the two main causes of forced terminations. 
- Propagation Impairments: These can be offset by reducing the fading losses and increasing the link margin. This can be easily done with a fixed terminal by selecting an appropriate site for the earth terminal with no surrounding obstacle and fitting the terminal with a sufficiently large antenna. However, such a solution is practically infeasible for mobile users. In this case, only a suitable link margin could overcome propagation impairments.

- Handover Failure: This can be avoided by using the earth-fixed cell technique [2], [4], which is for future LEO multimedia satellite systems such as Teledesic, ${ }^{1}$ Skybridge (formerly Sativod) [8], and M-STAR (now integrated into the Celestri project) [10]. But if the satellite-fixed cell technique is considered, it becomes necessary to implement a suitable channel reservation procedure that guarantees a call in progress the success of all its handovers. That service will be hereafter called guaranteed handover $(\mathrm{GH})$ service. Users having subscribed to this service will be named GH users, and their calls named GH calls while other users will be named regular users and their calls named regular calls. This GH service could appeal to business users who need to communicate from any region (even underdeveloped countries or remote regions on the earth) by means of a high-quality service. Moreover, the GH service could be exploited as a backup service for high-quality communications rerouted from the terrestrial cellular coverage. All these aspects concerning high-QoS mobile communications are particularly relevant in view of the UMTS scenario incorporating a satellite component.

Consequently, it makes sense to consider that non-GEO systems acting as satellite components of UMTS will simultaneously service two types of users (i.e., regular users and $\mathrm{GH}$ users), each category being treated through different contracts stipulating specific QoS. Hence, it is important to evaluate the impact on satellite capacity of servicing a mixed population of $\mathrm{GH}$ and regular users.

This paper focuses on LEO systems only since those systems are characterized by a higher handover rate than MEO systems and are therefore more sensitive to handover failure. A simple method to guarantee the success of any GH call handover would consist in reserving at call setup a channel in all the cells the user will visit. However, the duration of the call is not necessarily known at call setup, and the number of concerned cells is consequently uncertain. Moreover, this method leads to overdimensioning the satellite capacity, as the reserved channels would remain unused most of the time. Therefore, a more efficient channel reservation procedure should only reserve the strictly necessary capacity, during the minimum time, and as late as possible in the cells visited by the considered GH user. Such a channel reservation procedure, called channel-locking mechanism, is proposed hereafter. With such a procedure, the GH service can be offered to any user, be it fixed or mobile. The only requirement is that the user position be known at call setup and, for a mobile user, that the user speed be significantly lower than that of the subsatellite

\footnotetext{
${ }^{1}$ Visit http://www.teledesic.com
}

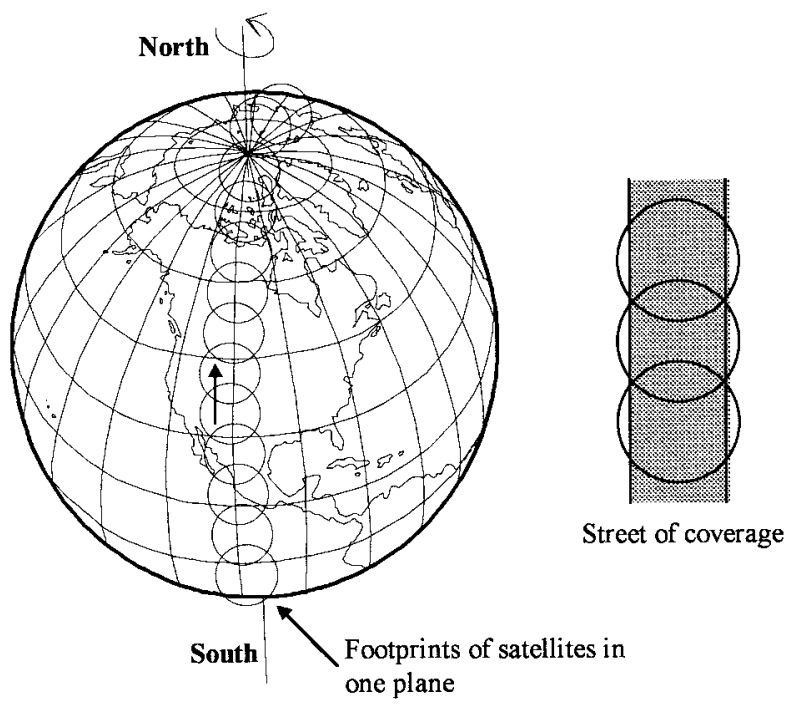

Fig. 1. Example of street of coverage (polar constellation).

point (typically, no more than a few hundred meters per second for a user serviced by an LEO satellite system). It is assumed that the user position can be determined by a positioning system integrated into the satellite system [9] at the call setup demand time with a sufficient accuracy.

The organization of the paper is as follows. Section II describes the cellular structure, mobility model, and proposed channel reservation procedure which guarantees $\mathrm{GH}$ users against handover failure. Section III presents the analytical approach used for performance evaluation. Section IV compares simulation and analytical results in terms of the probability of finding all channels busy in a cell (for regular users, this is both the blocking probability for new call attempts and the handover failure probability; for GH users, this probability is related to the blocking probability for new call attempts, as explained in Section III). Section V evaluates the capacity increase needed for the GH service and compares the performance of the GH scheme with that of the strategy based on queuing of handover requests for a portion of privileged users. Finally, Section VI summarizes the results and concludes.

\section{Channel Reservation Procedure FOR GUARANTEED HANDOVERS}

\section{A. Coverage Geometry}

The proposed channel reservation procedure is based on the existence of a street of coverage formed by all satellites participating in the sequence of successive handovers during a given call. The "street of coverage" concept applied to the satellite coverage refers to the region of the earth in form of a strip wherein contiguous coverage is ensured by several satellites in a given constellation [11]. A simple example of street of coverage is shown in Fig. 1 for a polar constellation, where all involved satellites are in a given plane.

The footprints of two successive satellites $S_{1}$ and $S_{2}$ can be extracted from Fig. 1 and are shown in Fig. 2, with details of the cellular coverage. Each satellite footprint incorporates overlapping adjacent cells. Cells are organized in tiers, each 


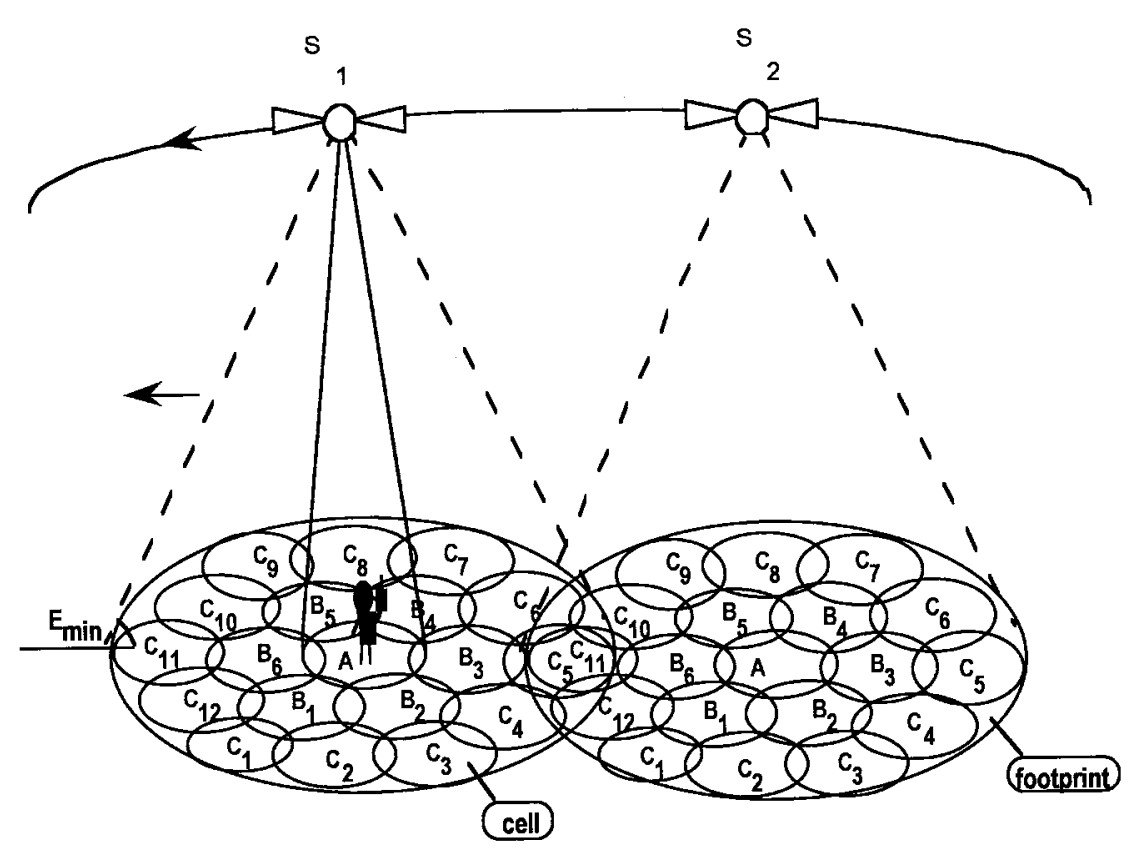

Fig. 2. Satellite coverage geometry.

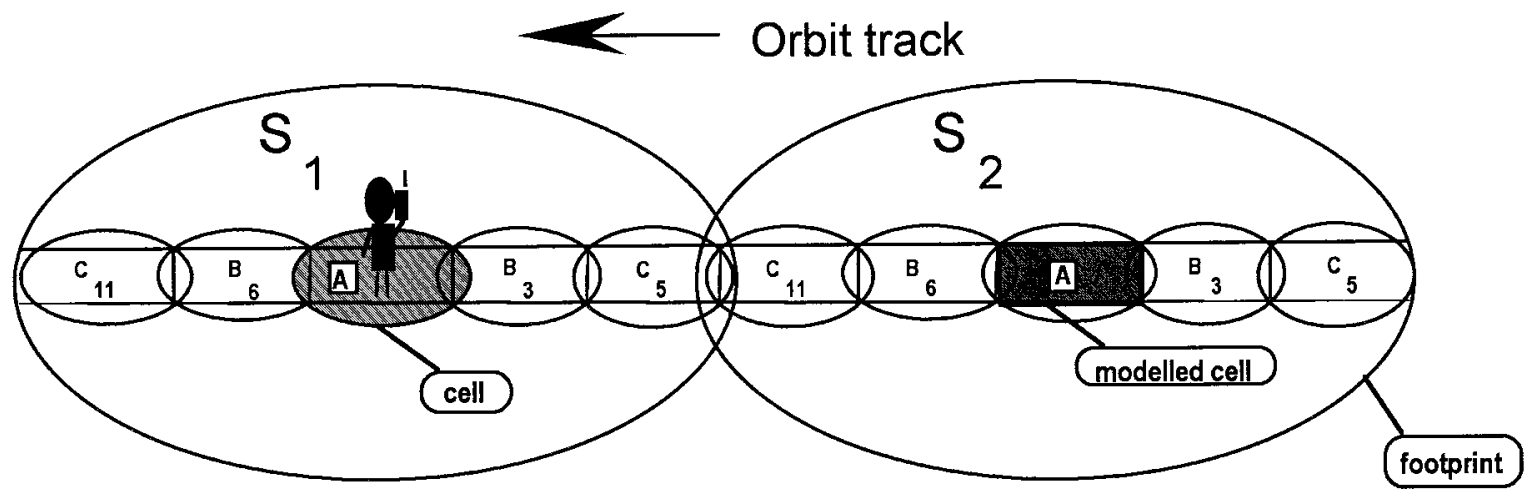

Fig. 3. Cell model.

tier encircling the inner one. Tiers are identified by letters $(A, B, C, \cdots)$ and cells in a given tier by subindex numbers $(1,2,3, \cdots)$. At the considered time, the user is located at the center of the area covered by satellite $S_{1}$. As satellite $S_{1}$ in Fig. 2 moves toward the left, the user passes from one cell to the next one on the right, within the footprint of satellite $S_{1}$. This is a beam handover. Eventually, the user will enter the overlap area between satellite $S_{1}$ and $S_{2}$ and will experience a satellite handover.

Both the rotation of the earth and the user speed are neglected with respect to the speed of the subsatellite points on the earth $V_{\text {trk }}$. Therefore, mobile and fixed users are treated similarly with respect to the handover occurrence, as shown in Section II-C.

\section{B. Cell Model}

The street of coverage concept also applies to a set of contiguous cells as long as some overlap exists between satellite footprints [11], as verified through orbit analyzer tools [12]. This is illustrated in Fig. 3, which is an excerpt of Fig. 2.
Each cell is modeled as a rectangle bounded by the parallel sides of a strip within the street of satellite coverage and by the segments joining the intersections of the circular boundaries of the cells. In the following, the cells in the strip within the street of coverage shown in Fig. 3 will be renumbered in sequence for commodity, i.e., $(i),(i+1),(i+2)$, etc.

The time interval to cross a cell $(i)$ from border to border, called user sojourn time in a cell $(i), T_{c}(i)$, is equal to the cell passage time and is given by

$$
T_{C}(i)=T_{\text {orb }} \frac{L(i)}{2 \pi R_{e}}
$$

where

$T_{\text {orb }} \quad$ orbit period;

$L(i) \quad$ length along the satellite track of the modeled cell (i) $[\mathrm{km}]$

$R_{e} \quad$ average radius of the earth $=6371 \mathrm{~km}$.

Since cells have a rectangular shape and users trajectories are parallel to the direction of their street of coverage, then $T_{c}(i)$ becomes a constant value, $T_{c}$, which is calculated 


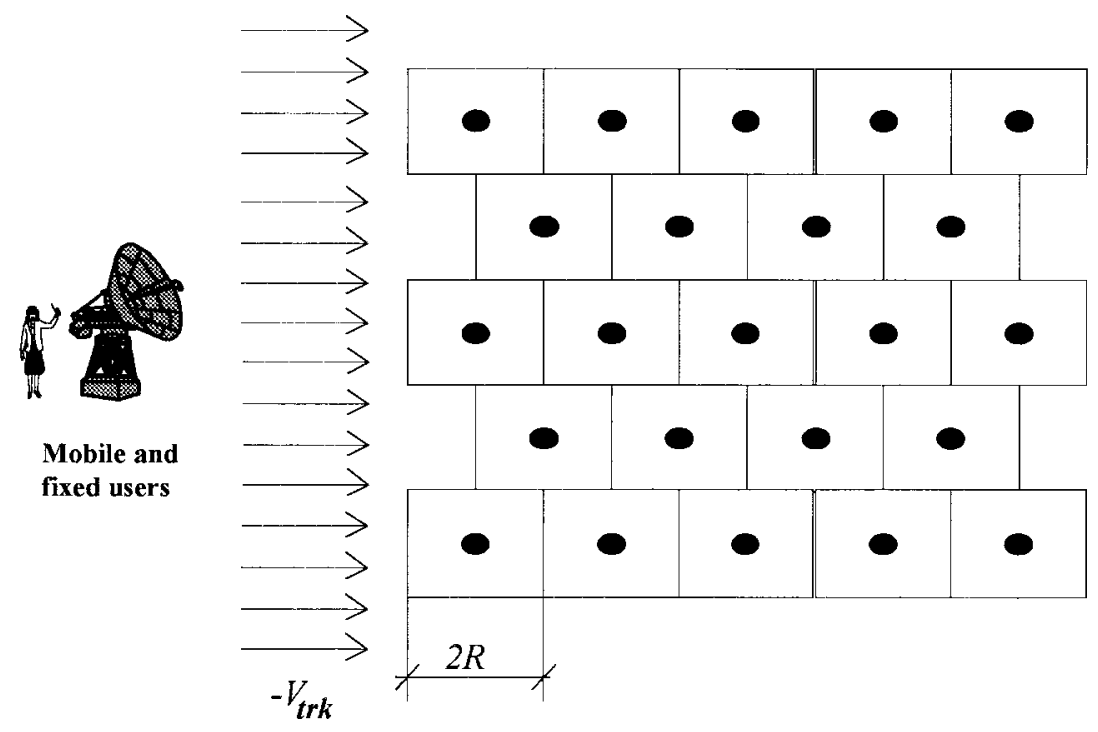

Fig. 4. Mobility model based on rectangular-shaped cells.

according to (1), putting $L(i)=2 R$, where $R$ is a constant value equal to one half the constant length of a cell (see Fig. 4).

\section{The Mobility Model}

With the cell configuration of Fig. 3 (i.e., street of coverage), one can apply the mobility model proposed in [13]-[15], which is illustrated in Fig. 4. This model is valid as far as the following assumptions are met.

1) When a handover occurs, the destination cell is the adjacent cell in the direction of the satellite subsatellite point motion. Users cross cell boundaries at a constant velocity aligned and opposite to the satellite subsatellite point speed, i.e., with velocity vector $-V_{\text {trk }}$. This assumption holds as far as the rotation of the earth (maximum speed at equator, $0.463 \mathrm{~km} / \mathrm{s}$ ) and the user speed (considered to be less than a few hundred $\mathrm{m} / \mathrm{s}$ ) are negligible with respect to the speed $V_{\text {trk }}$ of the subsatellite point on the earth, comprised between $7.1-5.3 \mathrm{~km} / \mathrm{s}$ for satellite altitudes from 500 to $2000 \mathrm{~km}$, respectively. These altitudes are the practical limits for LEO satellite systems. According to these considerations, user mobility is mainly due to the satellite constellation mobility.

2) From call setup, an active user travels a distance which is:

a) uniformly distributed between zero and $2 R$ for the source cell, which is the cell where the call is setup;

b) deterministically equal to $2 R$ for any transit cell, defined as any cell (subsequent to the source one) reached after call setup by the considered active user during call lifetime.

3) A handover procedure is initiated as soon as a active user with a call in progress reaches the boundary of an adjacent cell.

This mobility model is similar to that proposed in [16] for mobile cellular networks used in highways, except that now the mobile speed is no longer a random variable reflecting the variety of users, but has a constant value independent of the mobile speed and equal to the satellite ground-track speed $V_{\mathrm{trk}}=2 \pi R_{e} / T_{\mathrm{orb}}$.

\section{Reservation Procedure for the GH Service: \\ The Channel-Locking Mechanism}

A fixed channel allocation (FCA) scheme is considered for the allocation of satellite channels to beams [14]. Let us denote by $C(i)$ the number of channels assigned to cell $(i)$. GH user calls and regular user calls are treated differently both at call setup and at handover.

1) For regular user calls, setup and handover are managed similarly with no priority: a channel is allocated whenever available. If not, the call is blocked at setup or terminated at handover.

2) For GH user calls, setup and handover involve a specific reservation procedure, called here channel-locking mechanism, previously considered in [17]-[20]. ${ }^{2}$ It manages the first handover (the one immediately after GH user call setup) differently from subsequent handovers as follows.

a) Call Setup and First Handover: Since a GH user can be positioned at call setup anywhere in the source cell, the time interval from the instant of call setup to the instant of the first handover $T_{h 1}$ is a random variable within the interval $\left[0, T_{c}\right]$. Consequently, when the first handover occurs, the first transit cell may not dispose of an idle channel to serve the incoming call. To make sure that the first handover is successful, it is necessary to allocate two channels at GH call setup time: one to the source cell $(i)$ and another to the first transit cell $(i+1)$, thus, the channel reservation procedure is such that a new GH call originating in cell $(i)$ is accepted if and

\footnotetext{
${ }^{2}$ The main contribution of this work is to develop a performance analysis on the basis of a suitable mobility model for LEO-MSS's (see Section III). This study has been validated through simulations (see Section IV) and through the comparison with an alternative solution (see Section V).
} 
only if a channel is idle both in cell $(i)$ and in cell $(i+1)$. The channel allocated to cell $(i)$ is active during $T_{h 1}$, while the one allocated to cell $(i+1)$ is locked (although it is still idle). After $T_{h 1}$ (if the call is still active), the first handover occurs and the channel in cell $(i)$ is released. Simultaneously, one of the channels locked in cell $(i+1)$ is allocated to the handed-over GH call and remains active until the next handover or call completion, whichever first occurs. Should the call be completed within cell $(i)$, both the channel allocated to cell $(i)$ and a channel locked in the subsequent cell $(i+1)$ are released.

b) Subsequent Handovers: When the first handover occurs from cell $(i)$ to cell $(i+1)$, the call becomes served by one of the channels locked in cell $(i+$ 1 ), which now becomes active. Simultaneously, a new channel-locking request is issued for the next candidate transit cell $(i+2)$. Should any channel in cell $(i+2)$ be idle, a channel is immediately locked in that cell. Should all channels in cell $(i+2)$ be busy, the request is queued into a locking request list and will be satisfied according to a first-in first-out (FIFO) discipline. Then, channels released in cell $(i+2)$ are in priority used to serve the queued requests (if any). Hence, any new call (either GH or regular) or handed-over regular call will not be attended as long as a channel-locking request is in the queue. This procedure is repeated for each subsequent handover: at handover from cell $(i+1)$ to cell $(i+2)$, a channel-locking request is issued to cell $(i+3)$ and so on. However, subsequent handovers from cell $(i+n)$ to cell $(i+n+1)$ with $n>1$ may fail if the capacity (i.e., the number of channels) of the next transit cell $(i+n+1)$ is less than the maximum number of requests waiting in the queue. Therefore, the capacity $C(i)$ should be taken equal for all cells [i.e., $C(i)=C$ ] and evaluated so as to guarantee the required QoS in correspondence with peak traffic conditions (i.e., at time and location when and where the generation rate of new calls is at its highest level). Then, the total number of handed-over calls exiting any transit cell cannot exceed the channel capacity of the next transit cell. Therefore, the maximum size of the queue of the channel-locking requests in a cell is equal to or lower than $C$. Once a call experiences a handover from the source cell $(i)$ to the first transit cell $(i+1)$ at time $\tau_{0}=T_{o}+T_{h 1}$ (random handover), the subsequent handover from cell $(i+n)$ to cell $(i+n+1)$ with $n>0$, should it occur, will be at time $\tau_{n}=T_{o}+T_{h 1}+n T_{c}$ (periodic handover). ${ }^{3} \mathrm{At}$ time $\tau_{n}$, all calls in cell $(i+n+1)$ at the time of the previous handover from cell $(i+n-1)$ to cell $(i+n), \tau_{n-1}=T_{o}+T_{h 1}+(n-1) T_{c}$, have left cell $(i+n+1)$.

\footnotetext{
${ }^{3}$ The first handover is a random handover because it occurs at a random time after the call setup instant $T_{o}$, whereas subsequent handovers are periodic because they occur regularly after a time $T_{c}$.
}

The channel-locking mechanism for $\mathrm{GH}$ calls both at call setup and at handover is detailed in Fig. 5, which presents a group of $C$ users concentrated over a small area. In this situation (the worst case), two examples are considered to illustrate the need for the channel-locking mechanism for a GH call both at call setup [case a)] and at periodical handover [case b)]. Note that in Fig. 5, $\lambda_{G H n}$ is the rate of new GH call attempts, whereas $\lambda_{G H h}$ is the handover rate for GH calls.

- In case a), the new GH call attempt occurs in cell $(i)$ at time $T_{O}$ when there is a group of $C$ active users in the first transit cell $(i+1)$ in a position very close to the boundaries with cell $(i)$. Without any channel-locking mechanism, the first handover at time $T_{o}+T_{h 1}$ might be unsuccessful.

- In case b), a call entering the transit cell $(i)$ is positioned at the end of the queue for channel-locking requests in cell $(i+1)$ at time $\tau_{0}$. All other requests in cell $(i+1)$ will be satisfied within the time interval $T_{c}$ and the channellocking request from the considered call will become the first one in the queue. Now, the transit cell $(i+1)$ will release a channel at time $\tau_{1}$ at the latest, and the request will be satisfied on time.

From the above, it results that:

1) once a request is issued, the waiting time in the queue is lower than or equal to the time between subsequent handovers $T_{c}$;

2) the size of the waiting list for channel-locking requests is lower than or equal to the cell capacity $C$.

In conclusion, the channel-locking mechanism always guarantees a successful handover, while reserving the minimum capacity during the minimum time, thanks to the two following features:

- channel locking at call setup time only in the first transit cell;

- channel-locking requests for periodic handovers placed in the queue of the relevant transit cells (requests issued one cell in advance and no more than one cell).

\section{The Proposed AnAlytic ApProach}

\section{A. Basic Assumptions}

The user sojourn time in a cell, $T_{c}$, is assumed to be constant. The same mean call duration $T_{\text {call }}$ applies to both $\mathrm{GH}$ and regular calls. The allocation of capacity to beams is according to FCA, and the channel capacity of each cell is a constant $C$.

We develop in this section an analytical model to study each cell. This is an exponential model based on a Markov chain of the $M / M / C / S$ type. This notation refers to the following: the first $M$ stands for Poisson arrival process; the second $M$ stands for exponentially distributed service time; $C$ denotes the number of channels per cell; and $S$ is the number of states of the system considering both requests in service (both channels in use and channels locked) and channel-locking requests waiting service and then placed on a suitable waiting list. This model requires the following assumptions. 

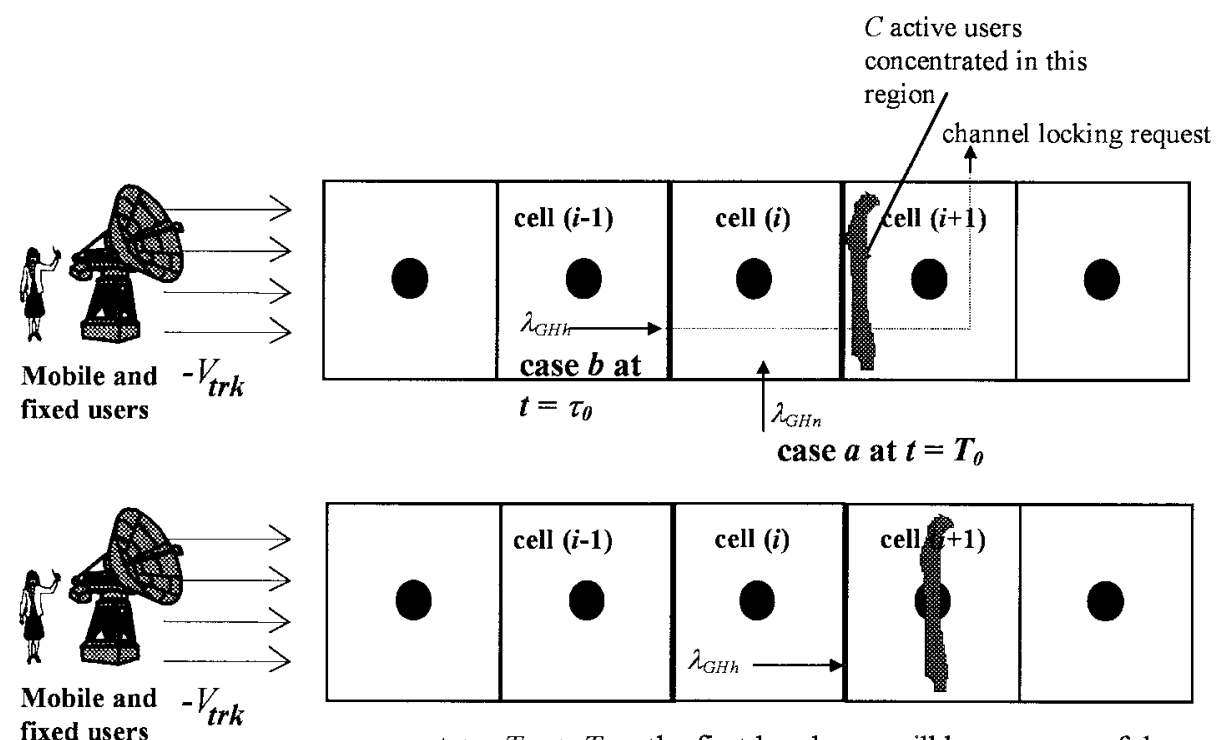
fixed users $t r k$

case $\boldsymbol{a}$ at $t=T_{0}+T_{h I}$ : the first handover will be unsuccessful with no channel locking in the first transit cell at call set-up.

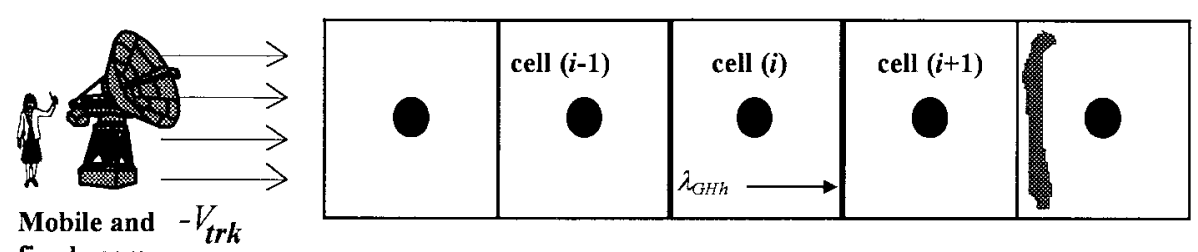

case $b$ at $t=\tau_{1}$ : subsequent handovers are successful thanks to the channel locking mechanism (one cell in advance).

Fig. 5. Necessity of a channel-locking mechanism for GH service. Case a): first handover (random handover) and case b): subsequent handovers (periodic handovers).

- The new call arrival processes for both regular and GH calls are independent Poisson processes. Therefore, this model considers an infinite population of users, but in practice (as considered in the simulations), the number of users is finite. Then, given the cell capacity and the call generation rate, the actual call blocking probability is lower than that obtained from the model [22]. Under specific considerations mentioned in Section IV, this approximation is acceptable.

- The handover arrival processes for both regular and GH calls are independent Poisson processes. This is a conservative assumption which results in overestimating the blocking probability, since the arrival processes for handed-over calls would be better modeled by smooth traffic $[21],{ }^{4}$ depending on the new call generation rates, the channel capacity of a cell, and user relative mobility (mainly, satellite constellation mobility).

- Exponentially distributed channel holding times in a cell: on the basis of the mobility assumptions, the channel holding time statistics are different for the source cell and the transit ones and do not follow an exponential dis-

${ }^{4}$ A smooth traffic gives a lower blocking probability than a Poisson one at a parity of system resources, average arrival rate, and service time distribution. The handover arrival process in a cell is not a Poisson process, but a smooth one because it can be thought of as the output traffic from the loss queuing systems that model either the cells in which the related call may be originated for GH calls or the cells from which the handover may be originated for regular calls. tribution. However, by applying the method (insensitivity property) described in [23] and [24], it is acceptable to approximate the actual distribution of the channel holding time by an exponential one with the same average value, as will be done in Section III-D. The good agreement obtained in Section IV between simulation results and analytic predictions will validate such an approximation.

- Finally, a uniform traffic per cell is considered for both regular and GH calls in the analytic model and in simulations. The traffic value corresponds to the peak value.

\section{B. Traffic Components in a Given Cell}

The arrival processes of channel requests in a cell are characterized by the following average rates:

$\lambda_{R n} \quad$ mean call generation rate of new regular calls;

$\lambda_{G H n}$ mean call generation rate of new GH calls;

$\lambda_{R h} \quad$ mean call generation rate of handed-over regular calls;

$\lambda_{G H h}$ mean call generation rate of handed-over GH calls.

Fig. 6 displays the different traffic components in need of a channel in a given cell. One can remark that a given cell will receive channel requests due to new GH call attempts generated by both users residing in the cell itself and in the preceding one. Fig. 6 also shows the channel-locking requests generated by handed-over GH calls (periodic handovers). 


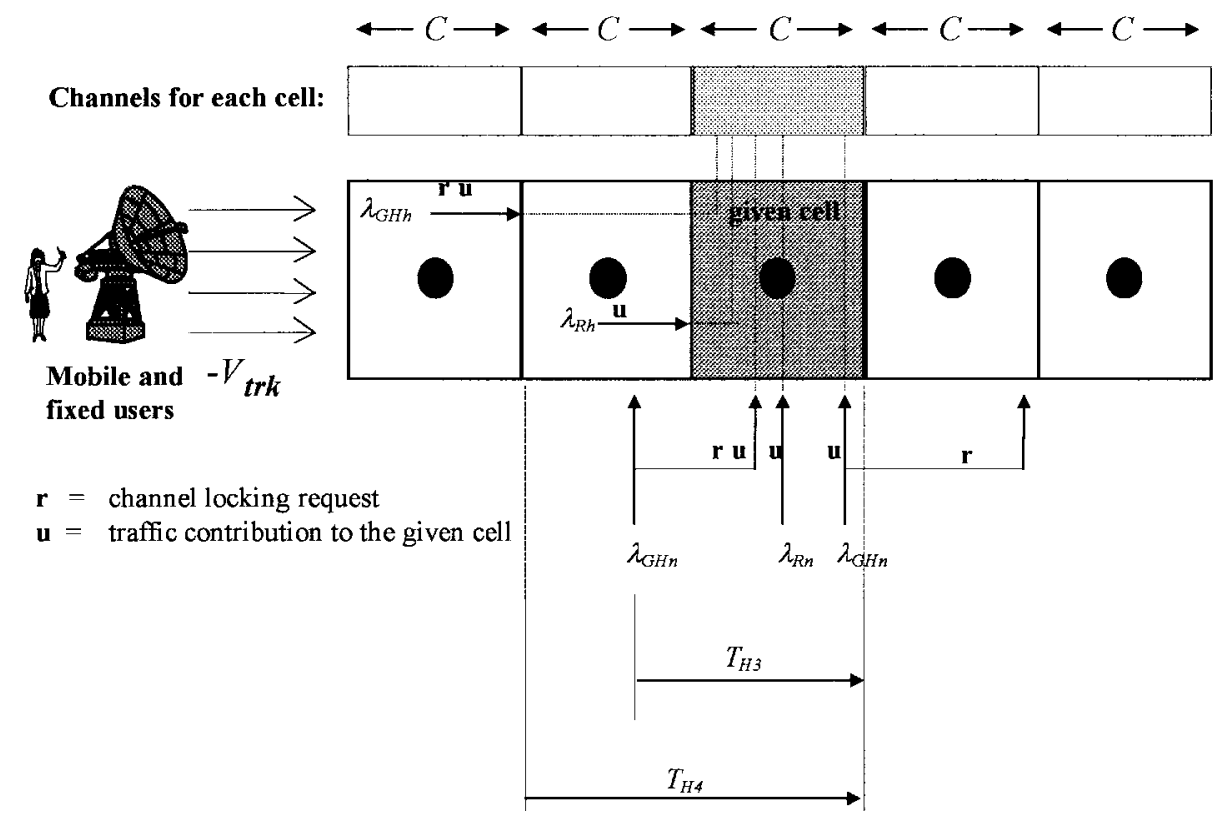

Fig. 6. Traffic contributions to a given cell from regular and GH calls.

Flux equilibrium for regular calls:

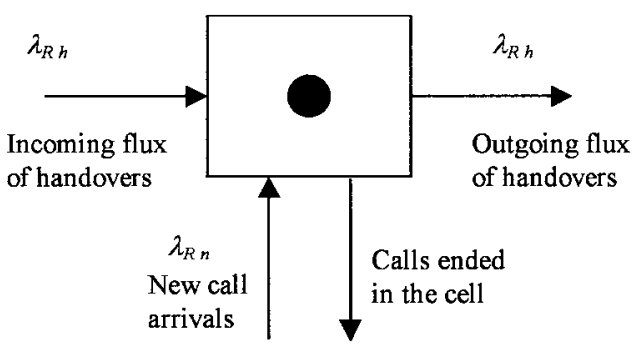

Fig. 7. Flux equilibrium conditions both regular and GH calls.

\section{Analysis of the Mobility Model}

Constellation mobility is characterized by a dimensionless parameter $\gamma$ defined as the ratio between the mean call duration and the user sojourn time in a cell (note that $\gamma$ is the reciprocal of parameter $\gamma$ introduced in [13]-[15])

$$
\gamma=\frac{T_{\text {cell }}}{T_{c}}
$$

where

$T_{c} \quad$ user sojourn time in a cell, which is equal to the cell passage time. According to (1), $T_{c}$ is given by

$$
T_{c}=2 R T_{\mathrm{orb}} /\left(2 \pi R_{e}\right)=2 R / V_{\mathrm{trk}}
$$

$T_{\text {call }}$ mean call duration for both regular and GH calls.

The handover probability depends on parameter $\gamma$ [14]. This probability is different for the first handover and the subsequent ones, because of the different distances covered by a user in the source cell and in the transit ones. The handover probability for a call in the source cell $P_{h 1}$ is given by

$$
P_{h 1}=\gamma\left(1-e^{-(1 / \gamma)}\right)
$$

Flux equilibrium for GH calls:

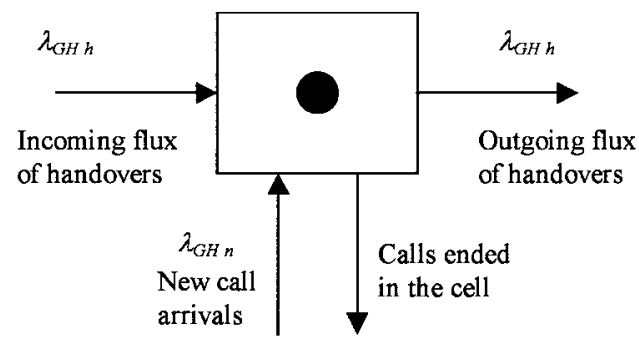

The handover probability for a call in a transit cell $P_{h 2}$ is given by

$$
P_{h 2}=e^{-(1 / \gamma)}
$$

As $\gamma$ increases, handover probabilities increase, thus also mobility increases. On the basis of [13]-[15], parameter $\gamma$ represents the average number of handover requests per call under the condition that there is no blocking (i.e., all channel requests are accepted by the system).

In order to derive the expression of the handover request arrival rate, as a function of both the new call arrival rate and the blocking probability $P_{b}$ (i.e., the probability that a channel request finds all channels busy in a cell), the handover requests are assumed to arrive in a cell according to a Poisson process, independent of the new call arrival process, ${ }^{5}$ and subjected to the condition of flux equilibrium in a cell between incoming and outgoing handovers [14]. This equilibrium condition will be separately applied for regular calls and GH ones, as illustrated in Fig. 7. Therefore, one has the following equilibrium conditions for regular and $\mathrm{GH}$ calls,

\footnotetext{
${ }^{5}$ This is an approximation commonly considered in the literature [13]; it can be considered acceptable for low values of $P_{b}$. In practice, this constraint is realistic if users must be provided with an acceptable QoS.
} 
respectively:

$$
\begin{gathered}
\lambda_{R h}\left(1-P_{b}\right) P_{h 2}+\lambda_{R n}\left(1-P_{b}\right) P_{h 1} \\
=\lambda_{R h}, \quad \text { for regular calls } \\
\lambda_{G H h} P_{h 2}+\lambda_{G H n}\left(1-P_{b}\right)^{2} P_{h 1} \\
=\lambda_{G H h}, \quad \text { for } \mathrm{GH} \text { calls. }
\end{gathered}
$$

For regular users, the equilibrium expression is the one derived in [14]. For GH calls, two main considerations hold.

- A new call attempt (mean rate $\lambda_{G H n}$ ) is accepted only if a channel is idle both in its source cell and in the first transit one. As all cells are identical and have the same traffic parameters, the probability of finding simultaneously an idle channel in each one of the two different cells is expressed by the factor $\left(1-P_{b}\right)^{2}$ which represents the connection probability for new GH call attempts, with the consequence that the blocking probability for new GH call attempts is equal to $2 P_{b}-P_{b}^{2}$.

- Handed-over calls (mean rate $\lambda_{G H h}$ ) never are blocked; then, the probability of success for each periodic handover is equal to one; so the connection probability is equal to one in the first term of (6), which is related to handed-over GH calls.

From the above relationships, the handover call arrival rates are given by

$$
\begin{aligned}
\lambda_{R h} & =\lambda_{R n} \frac{\left(1-P_{b}\right) P_{h 1}}{1-\left(1-P_{b}\right) P_{h 2}} \\
\lambda_{G H h} & =\lambda_{G H n} \frac{\left(1-P_{b}\right)^{2} P_{h 1}}{1-P_{h 2}}=\lambda_{G H n} \gamma\left(1-P_{b}\right)^{2} .
\end{aligned}
$$

\section{Traffic Intensity Offered to a Cell}

The derivation of the channel holding time in a cell by an exponential distribution, with rate $\mu$, follows that of [23] and [24]. One considers the mean channel holding time in a cell for different types of calls; subsequently, each average value is weighted with its occurrence probability; then, these contributions are summed so as to obtain the average channel holding time $1 / \mu$.

The following definitions are relevant:

- average value of the channel holding time in the source cell for both a regular call and a GH call: $E\left[T_{H 1}\right]$;

- average value of the channel holding time for a regular call in transit cells: $E\left[T_{H 2}\right]$;

- average value of the channel-locking time for a GH call in the first transit cell: $E\left[T_{H 3}\right]$;

- average value of the channel-locking time for a GH call in any transit cell other than the first one: $E\left[T_{H 4}\right]$.

Locking times as well as different traffic contributions to a given cell are illustrated in Fig. 6. Note that locking times incorporate both standby and active periods. The mean channel holding time in a cell, $1 / \mu$, is given by

$$
\begin{aligned}
\frac{1}{\mu}= & P_{1} E\left[T_{H 1}\right]+P_{2} E\left[T_{H 2}\right]+P_{3} E\left[T_{H 1}\right] \\
& +P_{3} E\left[T_{H 3}\right]+P_{4} E\left[T_{H 4}\right]
\end{aligned}
$$

where

$P_{1} \quad$ probability that a channel is occupied by a new regular call;

$P_{2} \quad$ probability that a channel is occupied by a handedover regular call;

$P_{3} \quad$ probability that a channel is occupied/locked by a new GH call in the considered cell/from the preceding cell;

$P_{4} \quad$ probability that a channel is locked by a handed-over GH call (arriving from one cell before the given cell).

$P_{1}, P_{2}, P_{3}$, and $P_{4}$ are given by

$$
\begin{aligned}
& P_{1}=\frac{\lambda_{R n}\left(1-P_{b}\right)}{\Lambda} \quad P_{2}=\frac{\lambda_{R h}\left(1-P_{b}\right)}{\Lambda} \\
& P_{3}=\frac{\lambda_{G H n}\left(1-P_{b}\right)^{2}}{\Lambda} \quad P_{4}=\frac{\lambda_{G H h}}{\Lambda}
\end{aligned}
$$

where $\lambda$ represents the mean rate of the total carried traffic and is given by

$$
\begin{aligned}
\Lambda= & \lambda_{R n}\left(1-P_{b}\right)+\lambda_{R h}\left(1-P_{b}\right)+\lambda_{G H n}\left(1-P_{b}\right)^{2} \\
& +\lambda_{G H n}\left(1-P_{b}\right)^{2}+\lambda_{G H n} .
\end{aligned}
$$

In (11), we have considered five contributions to $\Lambda$, each related to a different input traffic to a given cell, as shown in Fig. 6; in particular, each term is obtained as the product between one input mean generation rate and its related connection probability. Note that we have two contributions related to new GH calls (mean rate $\lambda_{G H n}$ ): that originated in the given cell, and the other originated in the preceding one. Both of them require that a channel be idle in two cells, and this explains the squared exponent over the probability of finding at least a free channel in a cell $\left(1-P_{b}\right)$. As handed-over GH calls (mean rate $\lambda_{G H h}$ ) never are blocked, their connection probability is equal to one.

The mean holding times $E\left[T_{H i}\right]$ are given by

$$
\begin{aligned}
& E\left[T_{H 1}\right]=T_{\text {call }}\left[1-P_{h 1}\right], \quad E\left[T_{H 2}\right]=T_{\text {call }}\left[1-P_{h 2}\right] \\
& E\left[T_{H 3}\right]=T_{\text {call }}\left[1-P_{h 1} P_{h 2}\right], \quad E\left[T_{H 4}\right]=T_{\text {call }}\left[1-\left(P_{h 2}\right)^{2}\right] .
\end{aligned}
$$

In particular, the expressions for $E\left[T_{H 1}\right]$ and $E\left[T_{H 2}\right]$ are the same as those derived in [14]. However, the expression for $E\left[T_{H 3}\right]$ considers the time spent both in the source cell and in the first transit one; the product $P_{h 1} P_{h 2}$ represents the probability that a GH user with a call in progress crosses both the source cell and the first transit one [25]. Similarly, the expression for $E\left[T_{H 4}\right]$ considers the time spent in two adjacent transit cells, and the factor $P_{h 2}^{2}$ represents the probability that a GH user call travels through two adjacent transit cells [25].

The total mean call arrival rate in a cell, $\lambda_{t}$, is defined as the sum of five different types of average arrival rates as follows:

$\lambda_{t}=\lambda_{R n}+\lambda_{R h}+\lambda_{G H n}\left(1-P_{b}\right)+\lambda_{G H n}\left(1-P_{b}\right)+\lambda_{G H h}$.

As in (11), this expression takes into account the two contributions related to new GH calls: that originated in the given cell, and the other originated in the preceding one. However, the existence of each one is conditioned by having an idle 
channel in the preceding cell for one of the contributions and in the following cell for the other. This justifies the use of the factor $\left(1-P_{b}\right)$ in both cases.

Finally, the total traffic intensity per cell, $\rho_{t}$, is given by

$$
\rho_{t}=\frac{\lambda_{t}}{\mu} \quad \text { (erlang). }
$$

Therefore, $\rho_{t}$ depends on both $P_{b}$ and $\gamma$. As the handed-over traffic is not an external variable [it depends on the new calls traffic, blocking conditions, and user mobility, as shown in (7) and (8)], it is convenient to define a mean generation rate per cell due to new calls only (both GH and regular), $\lambda_{t n}$, computed as follows:

$$
\lambda_{t n}=\lambda_{R n}+\lambda_{G H n} .
$$

In order to investigate the impact of the fraction of GH calls on system performance, a new parameter is now introduced $\left(k_{G H}\right)$, defined as the ratio of the mean generation rate for GH new calls $\left(\lambda_{G H n}\right)$ to the total mean generation rate per cell due to new calls $\left(\lambda_{t n}\right)$

$$
k_{G H}=\frac{\lambda_{G H n}}{\lambda_{t n}} .
$$

Note that $k_{G H}$ can be also considered as the portion of GH users among all the users. According to (16), the mean rates for both regular call attempts, $\lambda_{R n}$ and GH ones $\lambda_{G H n}$, can be defined with respect to $\lambda_{t n}$

$$
\lambda_{G H n}=\lambda_{t n} k_{G H} \quad \lambda_{R n}=\lambda_{t n}\left(1-k_{G H}\right) .
$$

Now, the total traffic intensity per cell due to new calls is defined by

$$
\rho_{t n}=\lambda_{t n} T_{\text {cell }} \quad \text { (erlang). }
$$

The traffic intensity $\rho_{t n}$ and parameter $k_{G H}$ will be considered as the input variables for both the analytic model and simulation runs. Hence, on the basis of the mean call duration, one finds out $\lambda_{t n}$ by using (18). Then, the mean arrival rates for both new regular calls and new GH calls are calculated by using $\lambda_{t n}$ and parameter $k_{G H}$ according to (17).

\section{E. The Queuing of Channel-Locking Requests}

Fig. 8 illustrates the waiting list for channel-locking requests, where the input traffic is represented by handed-over GH calls $\lambda_{G H h}$. A request is queued only if it finds no channel available in the cell (i.e., $C$ channels busy). Fig. 8 also presents the completion rates according to the exponential model that will be discussed in Section III-F. A request in the queue is satisfied either because a channel has been released in the cell (in the model, this occurs according to an exponential time distribution with mean rate $C \mu$ ) or because a call in the queue is ended (in the model, this corresponds to an exponential time distribution with mean rate $n \mu_{\text {call }}$, where $\mu_{\text {call }}=1 / T_{\text {call }}$, if there are $n$-locking requests in the queue). These aspects will be taken into account in Section III-F, where a Markov chain approach is proposed. Recall that according to the channellocking mechanism exposed in Section II-D, the maximum capacity needed for the waiting list is $C$.

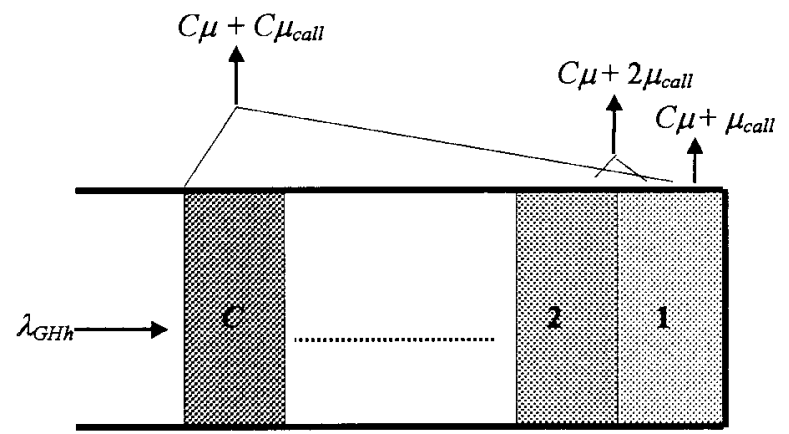

Fig. 8. The waiting list of channel-locking requests for handed-over GH users.

\section{F. A Markov Chain Approach}

From the above assumptions, it follows that each cell can be modeled as an M/M/C/S queuing system with nonhomogeneous arrival rates [26], [27]. The state of this queuing system is defined as the sum of the number of calls in service and the number of channel-locking requests for handed-over GH calls (both locking requests served and those in the waiting list). Parameter $S$ denotes the number of states of the queuing system. Since each cell has $C$ channels and the number of channel-locking requests can not exceed $C$, we have that $S=2 C$. The Markov chain model associated with each cell is shown in Fig. 9. This chain includes both the service and waiting parts due to the queuing of channellocking requests.

Whenever the system is in a state $n$, with $n$ smaller than $C$, the mean arrival rate is $\lambda_{t}$ as defined by (13) while, if the state corresponds to a value of $n$ greater than or equal to $C$, i.e., all channels in the cell are busy, the mean arrival rate is $\lambda_{G H h}$ (this refers to the channel-locking requests in the waiting list). When the system is in the state $C+i$ for $i=1,2, \cdots 2 C$, the contributions to the mean completion rate are (see Fig. 9):

- $C \mu$ due to the completion of the service of any of the calls in the cell (i.e., the holding time of a call in a cell is ended or a locking request issued to the cell has been cleared);

- $i \mu_{\text {call }}$ due to the clearing of a channel-locking request in the waiting list because the related call is ended before being served in the cell (i.e., before this call is actually handed over).

In particular, when there are $i$ channel-locking requests in the waiting list (the system is in the state $C+i$ ), the time to reach the state with $i-1$ channel-locking requests in the waiting list is exponentially distributed, and it is given by the minimum among $C$ (independent) channel holding times (each of them with an exponential distribution and mean rate $\left.\mu_{\text {call }}\right)$ and $i$ (independent) queue permanence times (each of them with an exponential distribution and mean rate $\left.\mu_{\text {call }}\right)$. Globally, this transition requires an exponentially distributed time with a mean rate equal to $C \mu+i \mu_{\text {call }}$ [27], [28], as considered in the waiting list shown in Fig. 8 and in the model shown in Fig. 9. 


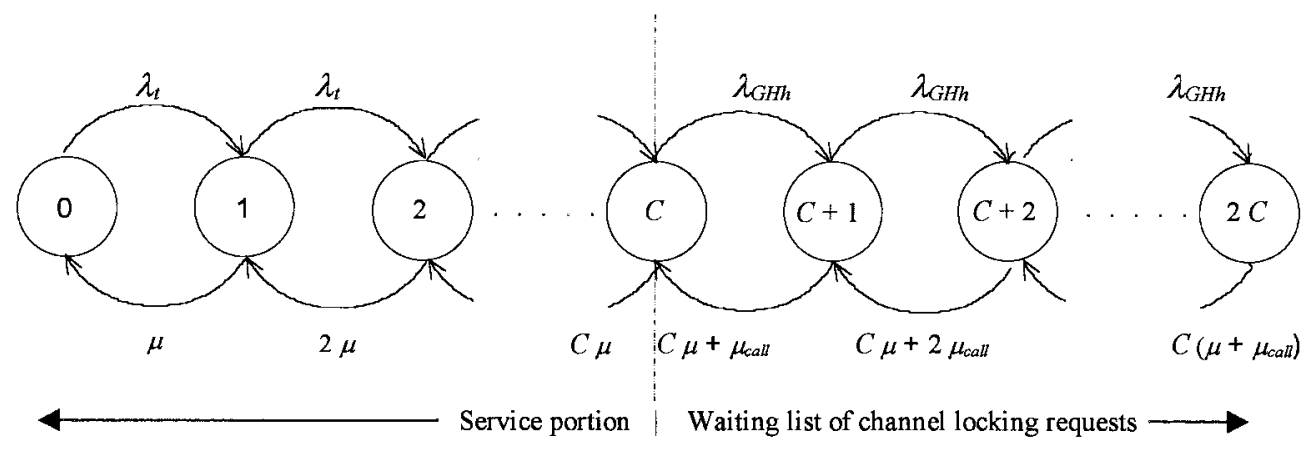

Fig. 9. Markov chain model for a cell with FCA.

By following the same approach as in [14] and [27], the probability of state $n, P_{n}$, can be derived as

$$
\frac{P_{n}}{P_{0}}= \begin{cases}\frac{\lambda_{t}^{n}}{n ! \mu^{n}}, & 1 \leq n \leq C-1 \\ \frac{\lambda_{t}^{C} \lambda_{G H h}^{n-C}}{C ! \mu^{C} \prod_{j=1}^{n-C}\left[C \mu+j \mu_{\text {call }}\right]}, & C \leq n \leq 2 C\end{cases}
$$

where the idle system probability $P_{0}$ is given by

$$
P_{0}=\left\{\sum_{n=0}^{C-1} \frac{\lambda_{t}^{n}}{n ! \mu^{n}}+\sum_{n=C}^{2 C} \frac{\lambda_{t}^{C} \lambda_{G H h}^{n-C}}{C ! \mu^{C} \prod_{j=1}^{n-C}\left[C \mu+j \mu_{\text {call }}\right]}\right\}^{-1} .
$$

The probability of finding all channels busy in a cell $P_{b}$ is given by

$$
P_{b}=\sum_{n=C}^{2 C} P_{n}
$$

A recursive approach is necessary to compute $P_{b}$, because $\mu$ and $\lambda_{t}$ depend on $P_{b}$ through (9) and (13), respectively. The system parameters are: $k_{G H}, C, \rho_{t n}, T_{\text {call }}$, and $\gamma$. The iterative method is based on parameter $P_{b}$ [13], [14]; we start the iterations with $P_{b}=0$ and we compute $\mu$ and $\lambda_{t}$; with these values, $P_{n}$ for $n=0,1, \cdots 2 C$ are computed according to (19) and (20). Then, according to (21) we obtain a new value for $P_{b}$. This value is averaged with that used at the previous step (i.e., zero at the first step). A new iteration starts with this average value of $P_{b}$. The iterative method is stopped when the relative difference between the $P_{b}$ values computed in two subsequent steps is below $10^{-3}$. Then, the value obtained for $P_{b}$ is used to derive the following different QoS parameters for both regular calls and $\mathrm{GH}$ ones.

For regular calls, since there is no handover prioritization, the blocking probability for new calls is equal to the handover failure probability and it is denoted as

$$
P_{b-R}=P_{b}
$$

According to [14] and [27], the probability for a regular call to be unsuccessful (due to either the initial blocking of the call attempt or the failure of a subsequent handover request) $P_{n s-R}$ is given by

$$
P_{n s-R}=P_{b}+\left(1-P_{b}\right) P_{\text {drop }-R}
$$

where $P_{\text {drop- }} R$ is the regular call forced-termination probability due to an unsuccessful handover, and it is given by

$$
P_{\text {drop }-R}=\frac{P_{b} P_{h 1}}{1-\left(1-P_{b}\right) P_{h 2}} .
$$

A new GH call is accepted if a channel is idle both in the source cell and in the first transit one. Therefore, the blocking probability of a new GH call $P_{b-G H}$ is given by

$$
P_{b-G H}=1-\left(1-P_{b}\right)^{2}=2 P_{b}-P_{b}^{2} .
$$

$\mathrm{GH}$ calls never experience handover failures, thanks to the channel-locking mechanism. Therefore, the call forcedtermination probability due to an unsuccessful handover for GH calls, $P_{\text {drop- } G H}$, is equal to zero

$$
P_{\text {drop }} G H=0 \text {. }
$$

Hence, the probability for a $\mathrm{GH}$ call to be unsuccessful, $P_{n s-G H}$, is given by

$$
P_{n s-G H}=P_{b-G H}+\left(1-P_{b-G H}\right) P_{\text {drop }-G H} \equiv P_{b-G H} .
$$

\section{Simulation AND AnAlytic Results}

Simulations have been carried out in order to validate the above analytic study. The simulated model is based on the following assumptions.

- The time to generate the next new call attempt on behalf of an inactive user is exponentially distributed with mean rate $\lambda_{\text {user }}$.

- The call duration is exponentially distributed with an average value $T_{\text {call }}=3 \mathrm{~min}$ (i.e., the usual value for telephone calls).

- A finite population of $U$ users per cell is assumed with $U \gg C$ (e.g., $U=100 C$ ) so as to reproduce the behavior of an infinite population of users; then, this validates the approximation of a Poisson arrival process for new call attempts.

- A uniform traffic density is considered.

- The quantity $U \lambda_{\text {user }} T_{\text {call }}$ used in the simulations corresponds to the traffic intensity $\rho_{t n}$ used in the theory.

- The mobility model with rectangular-shaped cells is used. 
The range of values for $\gamma$ depends on the size of the cell and the satellite velocity. Two different LEO scenarios have been considered:

- IRIDIUM-like ${ }^{6}$ : altitude $H=780 \mathrm{~km}$, cell length $L=$ $500 \mathrm{~km}$ (hence, $T_{c}=1.26 \mathrm{~min}$ and $\gamma=2.38$ ), traffic intensity per cell $\rho_{t n}$ from 1 to 8 erlangs, cell capacity $C=10$ channels, and $U=1000$ users per cell.

- GLOBALSTAR-like ${ }^{7}$ : altitude $H=1400 \mathrm{~km}$, cell length $L=1000 \mathrm{~km}$ (hence, $T_{c}=2.85 \mathrm{~min}$ and $\gamma=1.05$ ), traffic intensity per cell $\rho_{t n}$ from 5 to 16 erlangs, cell capacity $C=20$ channels, and $U=2000$ users per cell.

For these two LEO scenarios, Fig. 10(a) and (b) displays the blocking probability $P_{b}$ as a function of the total traffic intensity per cell due to new calls $\rho_{t n}$, respectively, for IRIDIUM-like and GLOBALSTAR-like cases. Note that $P_{b}$ is a significant QoS parameter which conditions all others QoS parameters (e.g., $P_{n s^{-} R}, P_{\text {drop- }}$, and $P_{n s-G H}$ ) as shown in the previous section; in particular, $P_{b}$ is both the blocking probability for regular call attempts and the handover failure probability for regular calls. In Fig. 10(a) and (b), $k_{G H}$ has been taken as a variable parameter in order to investigate the impact on QoS of varying the fraction of $\mathrm{GH}$ users within the total population of users. It can be seen that the blocking probability increases rapidly to unacceptable values as soon as the total load and/or the proportion of $\mathrm{GH}$ users becomes too high. A close agreement is shown to exist between the results obtained from the analytic model and the simulations.

\section{SATEllite CAPACity Design}

The goal of a service provider using an LEO constellation is to guarantee a QoS acceptable to customers. As seen above, providing the $\mathrm{GH}$ service to some users increases the blocking probability experienced by regular users which will not only suffer from an increased call setup blocking probability, but also from an increased handover failure probability. Hence, if an acceptable QoS has to be maintained for regular users, it is necessary to increase the channel capacity as the percentage of $\mathrm{GH}$ users augments. Let us consider a given requirement for the probability of an unsuccessful call for a regular user, $P_{n s-R}$. First of all, the model developed in this paper allows determining the cell capacity to fulfill this requirement when the system only services regular users (i.e., $k_{G H}=0$ ); this is the reference capacity $C\left(k_{G H}=0\right)$. Then, for nonzero values of $k_{G H}$, the percent capacity increase with respect to $C\left(k_{G H}=0\right)$ to fulfill the requirement for $P_{n s^{-}} \Delta C \%$ is evaluated

$$
\begin{gathered}
\Delta C\left(k_{G H}=X\right) \%=100\left[\frac{C\left(k_{G H}=X\right)}{C\left(k_{G H}=0\right)}-1\right] \\
\text { for } 0<X<100 .
\end{gathered}
$$

Fig. 11(a) and (b) relates, respectively, to the IRIDIUM-like case (i.e., $\gamma=2.38$ ) and to the GLOBALSTAR-like case (i.e., $\gamma=1.05)$. A target QoS requirement of $P_{n s-R}<2 \%$ has been retained, along with different traffic loads per cell (i.e., $2,4,6$, and 8 erlangs). The reference values of capacity,

\footnotetext{
${ }^{6}$ Visit http://www.iridium.com.

${ }^{7}$ Visit http://www.globalstar.com.
}

$C\left(k_{G H}=0\right)$, are given in Table I for every curve. Note that the slopes of the curves in Fig. 11(a) and (b) are not regular because these graphs have been obtained by evaluating the minimum number of channels that permits fulfilling the requirement on $P_{n s^{-}}$. Hence, the number of channels can only vary by integer quantities. In Fig. 11(a) and (b), this fact causes different slopes (including horizontal segments) for a given curve.

The first interesting result is the (large-scale) near-linear behavior of $\Delta C \%$ as a function of $k_{G H}$. Second, comparing Fig. 11(a) and (b), it can be observed that $\Delta C \%$ is not very sensitive to the mobility parameter $\gamma$, where $1<\gamma<2.5$; this range is relevant to LEO constellations.

Fig. 12(a) and (b) investigates the influence of selecting different QoS requirements for the probability of an unsuccessful call for a regular user $P_{n s^{-}}$. The required $\Delta C \%$ is shown as a function of $k_{G H}$ for the IRIDIUM-like constellation (i.e., $\gamma=2.38)$ selecting both a less severe and a more severe QoS requirement: $P_{n s-R}<5 \%$ and $P_{n s-R}<0.1 \%$, respectively. The reference values of capacity $C\left(k_{G H}=0\right)$ are also given in Table I. One can note that for a higher level of QoS (lower value of $P_{n s-R}$ ), the slope in the curves is not as pronounced as with a lower QoS.

As a rule of thumb, one can consider that $\Delta C \%$ is approximately a linear function of $k_{G H}$, such that

$$
\Delta C \%=K_{t} k_{G H}
$$

where $K_{t}$ is the slope of the curve which depends mostly on the required QoS and slightly on the traffic load and user mobility; practically, $\Delta C \%$ does not depend on the proportion of $\mathrm{GH}$ calls $k_{G H}$. In conclusion, we have

$$
K_{t}=K_{t}\left(\mathrm{QOS}, \rho_{t n}, \gamma\right)
$$

For instance, considering the IRIDIUM-like constellation, $K_{t} \approx 0.36$ for $P_{n s^{-} R}<0.1 \%, K_{t} \approx 0.47$ for $P_{n s^{-} R}<2 \%$, and $K_{t} \approx 0.53$ for $P_{n s-R}<5 \%$.

In order to validate the $\mathrm{GH}$ scheme proposed in this paper, we consider below the comparison with the channel reservation scheme proposed in [14]. In this alternative solution, two type of users are considered: regular users and privileged users (PU's). As for regular users, channel demands in a cell (i.e., both new call attempts and handover requests) are blocked and cleared when all channels are busy, whereas for privileged users, we consider a handover queuing scheme $(\mathrm{QH})$ in order to reduce the handover failure probability.

We assume that the portion of privileged users corresponds to that of GH users, which is denoted by $k_{G H}$. However, it is worth noting that, contrary to the $\mathrm{GH}$ scheme, the $\mathrm{QH}$ scheme does not avoid the risk of call dropping for privileged users.

The GH scheme and QH solution are compared by assuming the same mobility conditions, FCA with the same number of channels per cell, the same $k_{G H}$ value, and the same requirement for $P_{n s^{-}}$. In addition to this, in the $\mathrm{QH}$ solution we must consider an additional requirement on the calldropping probability for privileged users $P_{\text {drop- } P U}$.

Fig. 13(a) and (b) compares the $\mathrm{QH}$ scheme and the $\mathrm{GH}$ one in the IRIDIUM-like case and the GLOBALSAT-like one, 


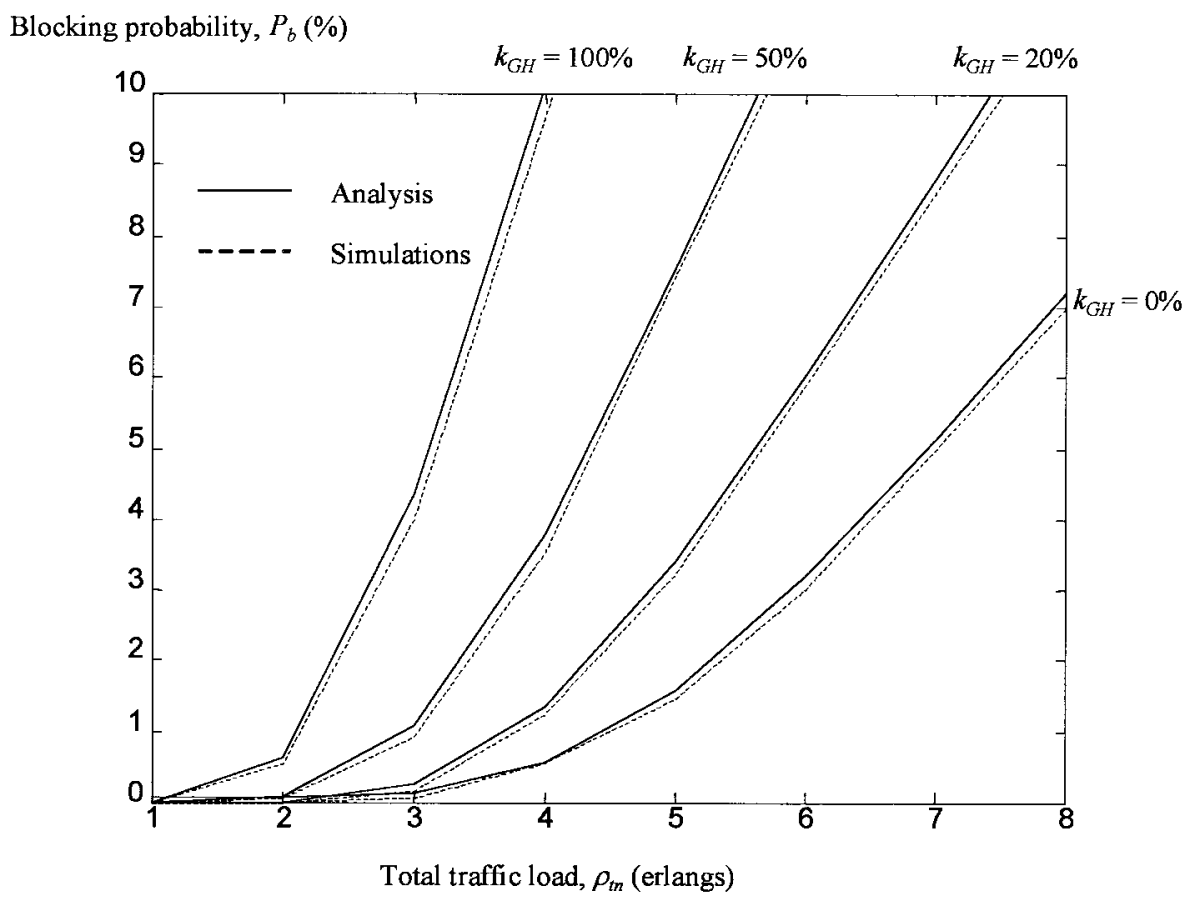

(a)

Blocking probability, $P_{b}(\%)$

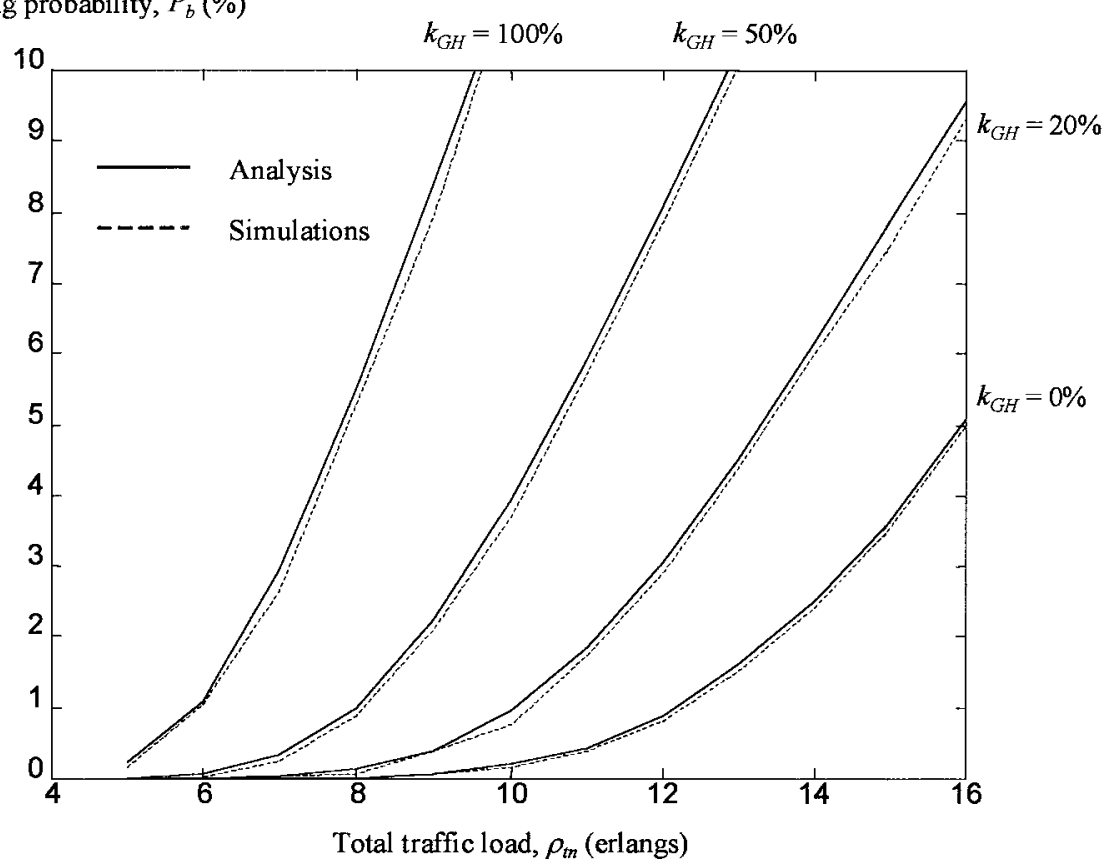

(b)

Fig. 10. (a) IRIDIUM-like constellation. Blocking probability $P_{b}$ versus total traffic load $\rho_{t n}$ for user mobility parameter $\gamma=2.38, C=10$ channels/cell, $U=1000$ users/cell, and different values of the portion of guaranteed handover users $k_{G H}$. (b) GLOBALSTAR-like constellation. Blocking probability $P_{b}$ versus total traffic load $\rho_{t n}$ for user mobility parameter $\gamma=1.05, C=20$ channels/cell, $U=2000$ users/cell, and different values of the portion of guaranteed handover users $k_{G H}$.

respectively, for $\rho_{t n}=8$ erlangs/cell in terms of the number of channels per cell (= cell capacity) to fulfill $P_{n s-R}<1 \%$ (for both the $\mathrm{QH}$ scheme and the $\mathrm{GH}$ one) and different requirements for $P_{\mathrm{drop}} \mathrm{PU}$ (only for the $\mathrm{QH}$ scheme). The results shown here for the $\mathrm{QH}$ solution have been obtained according to [14].

Note that the capacity per cell required in the QH scheme slightly depends on the $k_{G H}$ value. However, the GH scheme requires a lower number of channels per cell compared to the QH scheme from low values of $k_{G H}$ up to a crossover point which depends on the selected requirement for $P_{\text {drop}} P U$. In particular, if we consider $P_{\text {drop- } P U} \leq 0.01 \%$, the crossover occurs for $k_{G H}=0.3$ in the IRIDIUM-like case and for $k_{G H}=0.2$ in the GLOBALSTAR-like case. These $k_{G H}$ crossover values increase if stronger requirements are assumed for $P_{\mathrm{drop}}-P U$ (note that $P_{\mathrm{drop}}-G H=0$ ). Hence, these behav- 


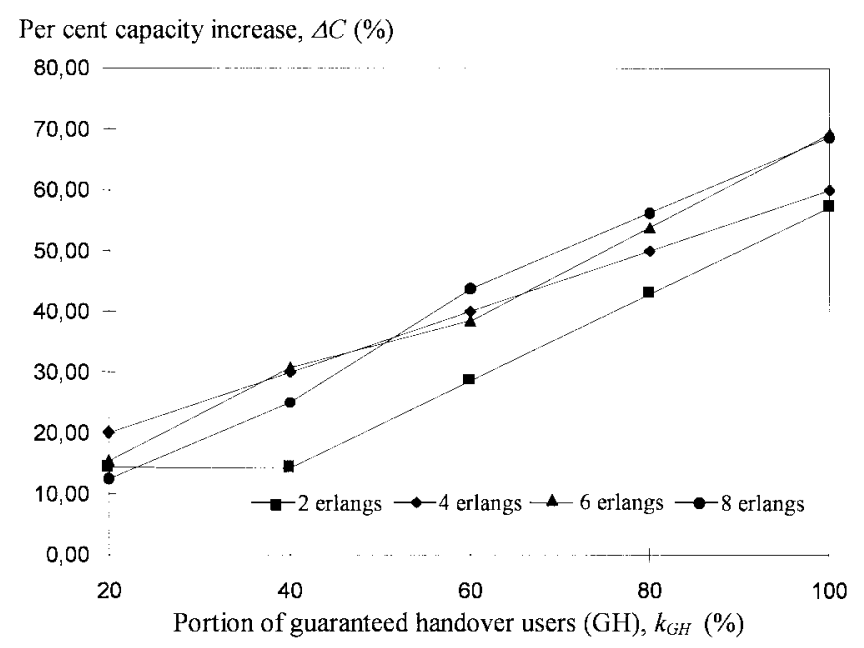

(a)

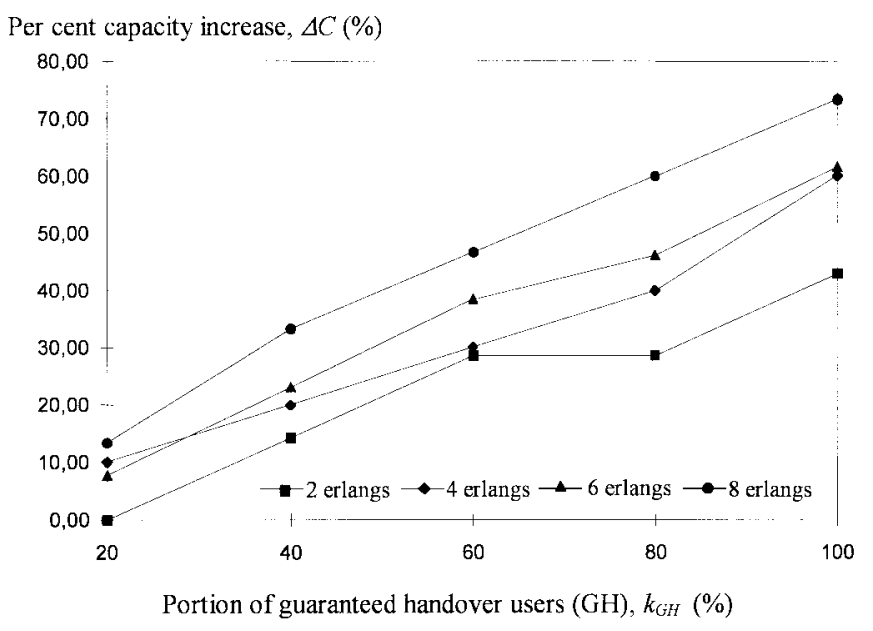

(b)

Fig. 11. (a) IRIDIUM-like constellation. $\Delta C \%$ versus $k_{G H}$ for $\gamma=2.38$ and $P_{n s-R}<2 \%$. (b) GLOBALSTAR-like constellation. $\Delta C \%$ versus $k_{G H}$ for $\gamma=1.05$ and $P_{n s-R}<2 \%$.

TABLE I

Cell Capacity Values for $k_{G H}=0$ and Different QoS Requirements for Regular Users

\begin{tabular}{c|c|c|c|c}
\hline $\begin{array}{c}\text { Traffic } \rho_{\text {tn }} \\
\text { (erlang) }\end{array}$ & $P_{n s-R}<2 \%, \gamma=2.38$ & $P_{n s-R}<2 \%, \gamma=1.05$ & $P_{n s-R}<0.1 \%, \gamma=2.38$ & $P_{n s-R}<5 \%, \gamma=2.38$ \\
\hline 2 & 7 & 7 & 9 & 6 \\
\hline 4 & 10 & 10 & 13 & 9 \\
\hline 6 & 13 & 12 & 17 & 12 \\
\hline 8 & 16 & 15 & 20 & 14 \\
\hline
\end{tabular}

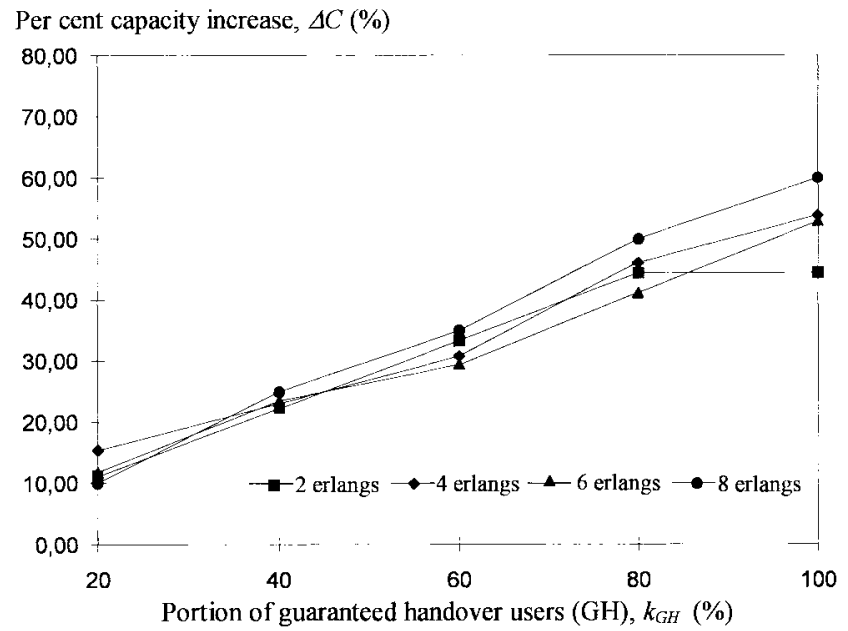

(a)

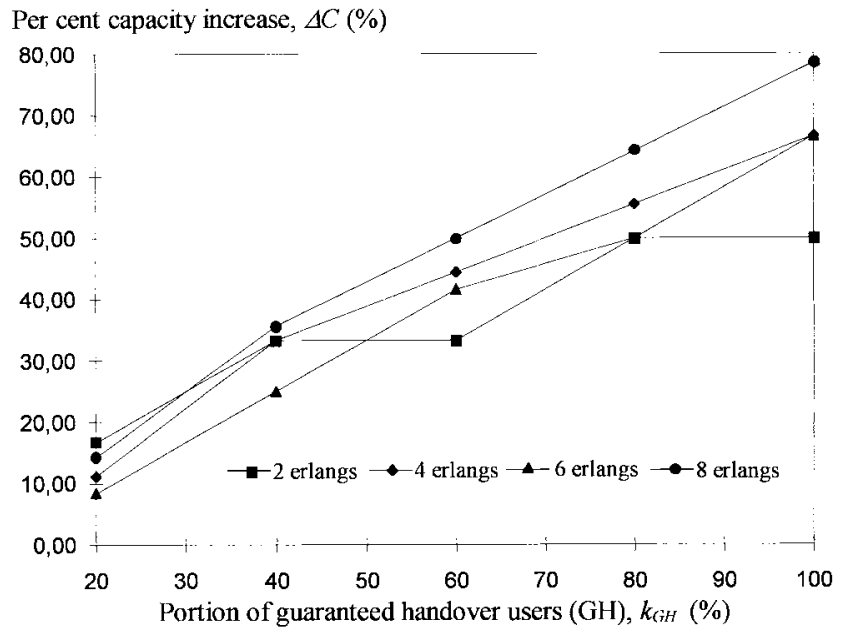

(b)

Fig. 12. (a) IRIDIUM-like constellation. $\Delta C \%$ versus $k_{G H}$ for $\gamma=2.38$ and $P_{n s-R}<0.1 \%$. (b) IRIDIUM-like constellation. $\Delta C \%$ versus $k_{G H}$ for $\gamma=2.38$ and $P_{n s-R}<5 \%$.

iors highlight that the GH scheme is convenient with respect to the $\mathrm{QH}$ one for low-to-medium $k_{G H}$ values.

\section{CONCLUSIONS}

A future UMTS scenario has been envisaged, where part of all the satellite component will be based on nongeostationary satellites. This mobile system will have a global coverage and should aim at providing the same QoS as for fixed terrestrial networks.

Toward this end, one of the most important aspects is the identification of suitable techniques to manage users' mobility. As a matter of fact, in nongeostationary satellite systems where cells are fixed with respect to the satellite, handover requests are frequent during call lifetime. Hence, 


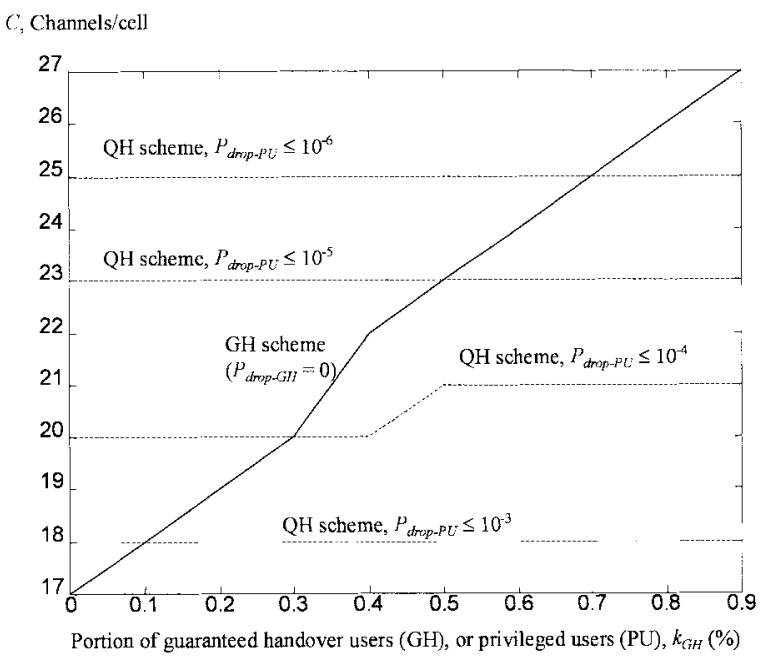

(a)

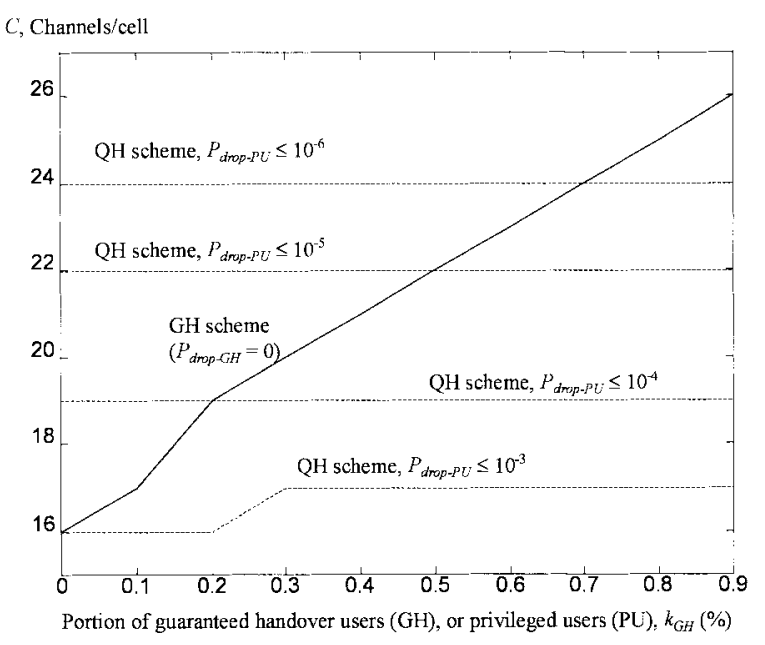

(b)

Fig. 13. (a) Comparison between the GH and QH schemes in terms of the number of channels for $P_{n s-R}<1 \%$ and several requirements for the call-dropping probability for privileged users $P_{\text {drop }-P U}$ (IRIDIUM-like constellation, $\gamma=2.38$ with $\rho_{t n}=8$ erlangs/cell). (b) Comparison between the GH and QH schemes in terms of the number of channels for $P_{n s-R}<1 \%$ and several requirements for the call-dropping probability for privileged users $P_{\mathrm{d}}$ rop-PU (GLOBALSTAR-like constellation, $\gamma=1.05$ with $\rho_{t n}=8$ erlangs/cell).

this paper has presented a procedure which guarantees successful handovers (called GH service) to candidate subscribers in an LEO constellation. Such a procedure assures a high QoS, analogous to that provided by terrestrial fixed networks.

An analytic approach has been developed to evaluate the impact of GH service subscription on system performance. This model has been successfully validated throughout the comparison with simulation results. It has been shown that providing the $\mathrm{GH}$ service to some users while assuring a given lower QoS for regular users requires an increased satellite capacity with respect to the case where all the users are served with the lower QoS. We have investigated the impact of parameters such as the constellation mobility, traffic load, and the percentage of $\mathrm{GH}$ calls on the per cent capacity increase $\Delta C \%$. A near-linear increase of $\Delta C \%$ has been shown to exist with respect to the percentage of $\mathrm{GH}$ users, with slope depending mostly on the required QoS, and slightly on the traffic load or the constellation mobility.

Finally, the capacity required for the $\mathrm{GH}$ scheme to guarantee a given QoS for regular users has been compared with that needed for an alternative solution based on the queuing of handover requests for privileged users. We have found that under moderate (and reasonable) percentages of $\mathrm{GH}$ users, the GH scheme attains a superior QoS than the queuing scheme and with a lower cell capacity.

We are confident that this analytical study will be helpful to the UMTS system designer in sizing the satellite capacity for a given population of $\mathrm{GH}$ users.

\section{ACKNOWLEDGMENT}

This work was carried out under joint cooperation between the Ecole Nationale Supérieure des Télécommunications (Site de Toulouse) and the University of Florence.

\section{REFERENCES}

[1] "Green paper on a common approach in the field of mobile and personal communications in the European Union," European Commission, 1994.

[2] J. Restrepo and G. Maral, "Coverage concepts for satellite constellations providing communications services to fixed and mobile users," Space Commun., vol. 13, no. 2, pp. 145-157, 1995.

[3] K. Johannsen, "Mobile P-service satellite system comparison," Int. J. Satell. Commun., vol. 13, no. 6, pp. 453-471, 1995.

[4] J. Restrepo and G. Maral, "Constellation sizing for non-GEO "earthfixed cell' satellite systems," in Proc. AIAA 16th Int. Communications Satellite Systems Conf. and Exhibit, Washington, DC, Feb. 25-29, 1996, pp. 768-778.

[5] F. Dosiere, T. Zein, and G. Maral, "A model for the handover traffic in low earth-orbiting (LEO) satellite networks for personal communications," Int. J. Satell. Commun., vol. 11, no. 6, pp. 145-149, 1993.

[6] S. Rappaport, "The multiple-call hand-off problem in high-capacity cellular communications systems," IEEE Trans. Veh. Technol., vol. 40, no. 3, pp. 546-557, 1991.

[7] ITU-T Recommendation E.771, "Network grade of service parameters and target values for circuit-switched land mobile services," Blue Book, 1995.

[8] A. Espace, "SATIVOD: A broadband multimedia access system using an innovative low earth orbiting satellite solution," Alcatel, June 1996.

[9] W. Zhao, R. Tafazolli, and B. G. Evans, "A UT positioning approach for dynamic satellite constellations," in Proc. 4th IMSC'95, Ottawa, Canada, June 6-8, 1995, pp. 251-258.

[10] Motorola Satellite Systems Inc., "Application for authority to construct, launch and operate the M-star systems," F.C.C., Washington, DC, Sept. 4, 1996.

[11] L. Rider, "Analytic design of satellite constellations for zonal earth coverage using inclined orbits," J. Astronautical Sci., pp. 31-64, Jan. 1986.

[12] J. J. Derrider, "LEONART: A low orbit satellite communication system analysis framework," in Proc. 4th Int. Conf. Telecommunications Syst., Nashville, TN, Mar. 21-24, 1996.

[13] E. Del Re, R. Fantacci, and G. Giambene, "Performance analysis of dynamic channel allocation technique for satellite mobile cellular networks," Int. J. Satell. Commun., vol. 12, no. 1, pp. 25-32, 1994.

[14] _ _Efficient dynamic channel allocation techniques with handover queuing for mobile satellite networks," IEEE J. Select. Areas Commun., vol. 13, no. 2, pp. 397-405, 1995.

[15] _ _ "An efficient technique for dynamically allocating channels in satellite cellular networks," in Proc. IEEE GLOBECOM '95, Singapore, Nov. 13-17, 1995, pp. 1624-1628.

[16] S. El-Dolil., "Teletraffic performance of highway microcells with overlay macrocell," IEEE J. Select. Areas Commun., vol. 7, no. 1, pp. 71-78, 1989. 
[17] J. Restrepo and G. Maral, "Providing appropriate grades of service to fixed and mobile users in a non-GEO satellite-fixed cell system," in Proc. 2nd European Workshop on Mobile/Personal Satcoms, Rome, Italy, Oct. 9-11, 1996.

[18] "Guaranteed handover (GH) service in a non-GEO constellation with "satellite-fixed cell" (SFC) systems," in Proc. NASA-JPL Int. Mobile Satellite Conf., Satcoms, IMSC '97, Pasadena, CA, June 16-18, 1997.

[19] J. Restrepo, "Comparative analysis between non-GEO satellite constellations (satellite-fixed cell and earth-fixed cell) servicing a mixed population of fixed and mobile users," Ph.D. dissertation, ENST, June 12, 1997.

[20] G. Maral and J. Restrepo, "Procede permettant de reduire la probabilité de blocage des appeles en provenance d'usagers à transfert garanti par prise en compte de la position relative des usafers," France Telecom Patent 97-02392, Feb. 26, 1997.

[21] A. A. Fredericks, "Congestion in blocking systems-A simple approximation technique," Bell Syst. Tech. J., vol. 59, no. 6, pp. 805-825, July-Aug. 1980.

[22] M. Schwartz, Telecommunication Networks: Protocols, Modeling and Analysis. New York: Addison-Wesley, 1987.

[23] D. K. Kim and D. K. Sung, "Handoff/resource managements based on PCV's and SVC's in broadband personal communication networks," in Proc. IEEE GLOBECOM '96, London, U.K., Nov. 18-22, 1996.

[24] K. W. Ross, Multiservice Loss Models for Broadband Telecommunication Networks. New York: Springer-Verlag, 1995.

[25] Y.-B. Lin, L.-F. Chang, and A. Noerpel, "Modeling hierarchical microcell/macrocell PCS architecture," in IEEE Proc. ICC'95, Seattle, June 1995, pp. 405-409.

[26] S. Tekinay and B. Jabbari, "A measurement-based prioritization scheme for handovers in mobile cellular networks," IEEE J. Select. Areas Commun., vol. 10, no. 8, pp. 1343-1350, 1992.

[27] D. Hong and S. S. Rappaport, "Traffic model and performance analysis for cellular mobile radio telephone systems with prioritized and nonprioritized handoff procedures," IEEE Trans. Veh. Technol., vol. VT-35, no. 3, pp. 77-92, 1986.

[28] L. Kleinrock, Queuing Systems. New York: Wiley, 1976.

Gérard Maral received the Doctorat d'Etat degree from the French University, Toulouse, France, in 1970.

He was appointed as Professor at the University of Toulouse, Toulouse, France. He later joined the Ecole Nationale Superieure des Telecommunications (ENST) in 1984 as a Full-Time Professor and subsequently developed the Toulouse of ENST site and its related satellite communications systems educational and research program. Presently, he heads the Toulouse of ENST site. He has 25 years of teaching and research experience in the field of electronics and communications and is a well-known international expert and lecturer on various aspects of satellite communications. He is a regular consultant to space industry and to the European Space Agency. He has authored articles in the areas of computer-aided design, digital communications, and advanced satellite communications systems and is the author of textbooks on satellite communications: Télécommunications par Satellite (Masson, 1982), Satellite Communications Systems (New York: Wiley, 1986, 1994, and 1998), and VSAT Networks (New York: Wiley, 1995). $\mathrm{He}$ is a Member of the Editorial Board of the International Journal of Satellite Communications, Space Communications, and Telecommunications Systems. He holds several patents in the field of satellite communications.

Dr. Maral is the Chairman of the COST253 action (service-efficient network interconnection via satellites).

Joaquin Restrepo received the degree in electronic engineering technological management from the Universidad Pontificia Bolivariana, Colombia, in 1992 and the Ph.D. degree from the Ecole Nationale Supérieure des Télécommunications (ENST), Toulouse, France, in 1997.

Currently, he is with the Universidad Pontificia Bolivariana, Formacion Avanzada. His research interests include mobile satellite systems and the cellular coverage of nongeostationary satellite constellations.
Enrico Del Re (M'78-SM'84) was born in Florence, Italy, in 1947. He received the Dr.Ing. degree in electronics engineering from the University of Pisa, Pisa, Italy, in 1971.

Until 1975, he worked in public administration and private firms, involved in the analysis and design of the telecommunication and air traffic control equipment and space systems. Since 1975, he has been with the Department of Electronics Engineering, University of Florence, Florence, Italy, first as a Research Assistant, then as an Associate Professor. Since 1986, he has been a Professor at the University of Florence. During the academic year 1987-1988, he was on leave from the University of Florence for a ninemonth period of research at the European Space Research and Technology Center of the European Space Agency, The Netherlands. He is the Head of the Digital Signal Processing and Telematics Laboratory (LENST), Department of Electronic Engineering, University of Florence. He also teaches a course in digital signal processing. His main research interests are digital signal processing, digital transmission techniques, and communication networks, on which he has published more than 150 papers in international journals and conferences. He is the coeditor of Satellite Integrated Communications Networks (North-Holland, 1988) and a coauthor of Data Compression and Error Control Techniques with Applications (New York: Academic, 1985).

Dr. Del Re has been the Chairman of the European Project COST 227 "Integrated space/terrestrial mobile networks" and is now Chairman of the COST 252 Project "Evolution of satellite personal communications from 2nd to future generation systems." He received the 1988/89 premium from the IEE (U.K.) for the paper "Multicarrier demodulator for digital satellite communication systems." He has been included in Who's Who in the World. $\mathrm{He}$ is a Member of the AEI and European Association for Signal Processing (EURASIP).

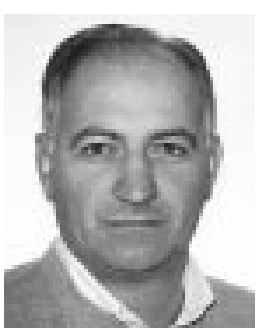

Romano Fantacci (S'82-M'87-SM'91) was born in Pistoia, Italy. He received the Dr.Ing. degree in electronics engineering in 1982 and the Ph.D. degree in telecommunication engineering in 1987, both from the University of Florence, Florence, Italy.

In 1982, he joined the Electronics Engineering Department, University of Florence as a Researcher in Computer Communications, where he is now an Associate Professor of Telecommunication Networks. During the first stage of his research activity, he worked on satellite communication systems. In particular, he has been involved in several European Space Agency (ESA) and INTELSAT advanced research projects. His present research interests involve digital communications, computer communications, queueing theory, fast packet switching, and mobile communication networks. He is the author of works that have been published in several journals in communication science.

Dr. Fantacci has been involved in several European (RACE) and nationwide research projects sponsored by the Italian National Council of Research (C.N.R.) and Italian Minister of Education and Research (MURST).

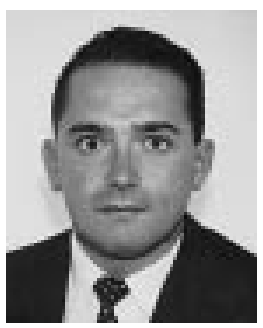

Giovanni Giambene (S'94-M'97) received the Dr.Ing. degree in electronics in 1993 and the Ph.D. degree in telecommunications and informatics in 1997, both from the University of Florence, Florence, Italy.

Since 1994, he has been with the Electronic Engineering Department, University of Florence, as a Research Assistant. His research interests include mobile cellular communication networks, personal communication services, channel allocation techniques, and queuing theory.

Dr. Giambene was the Technical External Secretary of the European Community Project COST 227 "Integrated space/terrestrial mobile networks." $\mathrm{He}$ also contributed to the "resource management" activity of Working Group 3000 within the RACE Project called "Satellite integration in the future mobile network" (SAINT, RACE 2117). 